\title{
PERFORMANCE ANALYSIS OF DS-CDMA SYSTEM FOR UNDERWATER ACOUSTIC COMMUNICATIONS
}

\author{
Haoxin Kang \\ Department of Electronic Information Engineering \\ North China University of Technology, Beijing, China
}

\begin{abstract}
We choose direct sequence spread spectrum (DSSS)technologies combined with Rake receiver under the modulation of BPSK to establish the communication modem. The performance of the modem is extensively investigated by MATLAB based simulation. We analysis the bit error rates (BER) against signal to noise ratio (SNR) corresponding to different parameter settings, for instance, under different Pseudo noise (PN)codes, the long of PN code period, the modulation schemes. Regarding to the Rake receiver, we also compare the scatter plots to deduce whether rake has better performance than without rake, furthermore we change the taps of the rake and diversity combining schemes to continue comparing the BER performance. The modem is implemented in a linear time invariant (LTI)channel model.
\end{abstract}

Keywords - Underwater acoustic, DSSS, Rake receiver, BPSK, LTI

\section{INTRODUCTION}

Underwater Acoustic Communication (UAC) play an important role in our life which serve us in various areas, such as military, environment monitoring, resource exploiting. The UAC environment is complex due to the dynamic channel, such as Doppler effect, marine life, variable temperature, pressure and etc. The light velocity in radio communication is $3 \times 10^{8} \mathrm{~m} / \mathrm{s}$, however the underwater sound velocity is approximately $1500 \mathrm{~m} / \mathrm{s}$ that is much slower than in radio communication [1], when the acoustic signal transmitted in the underwater channel, there exists large propagation delay, therefore employing acoustic waves to transmit data is becoming a more attractive way. The channel modelling of underwater communication can be regarded as a time-varying multipath channel, signal transmission here will be more challenging due to multipath effect and Doppler distortions. Rake receiver has a good performance to combat multipath effect because of its specific receiving algorithm.

In this paper, the characteristic of underwater channel is analyzed, and then what factors would interference the acoustic waves are introduced in section II. We provide a robust solution based on DSSS modulation schemes to combat the multipath fading of UAC channel, the related knowledge will present. The simulation results under different circumstances are presented in section III. Finally, followed by a conclusion in section IV.

\section{System Model}

\section{A. Attenuation -}

In UWA channels, the attenuation factor typically consist of absorption and scattering of the propagated acoustic wave [2]. Absorption related to the energy consumption from acoustic energy into heat, which increases not only depend on the transmission distance, but also with frequency, hence the bandwidth is limited [2]. The acoustic signals may also be attenuated by the process of scattering due to its direction is random, which happens from the ocean surface and bottom. However, in practice, we may not clearly distinguish the effects of absorption and scattering, and hence, we usually combined the two terms together [2]. The attenuation can be expressed as below equations [3]:

$$
A(l, f)=l^{k} a(f)^{l}
$$

where 1 is transmission distance, $\mathrm{f}$ is the frequency of propagated signal, $\mathrm{k}$ is the spreading factor(usually take 1.5 for practical [4]), which describes the propagation geometry. Thorp propose the mathematical way of expressing absorption coefficient [5] for interest frequency in $\mathrm{dB} / \mathrm{km}$ for $\mathrm{f}$ in $\mathrm{kHz}$ :

$$
\begin{gathered}
a(f)=0.11 \frac{f^{2}}{1+f^{2}}+44 \frac{f^{2}}{4100+f^{2}}+2.75 \cdot 10^{-4} f^{2} \\
+0.003
\end{gathered}
$$

Fig. 1 shows with the increasing of frequency, the path loss will also dramatically increase at the same time, therefore, in practice, we should choose a reasonable frequency to get the expected available bandwidth for UAC system. 


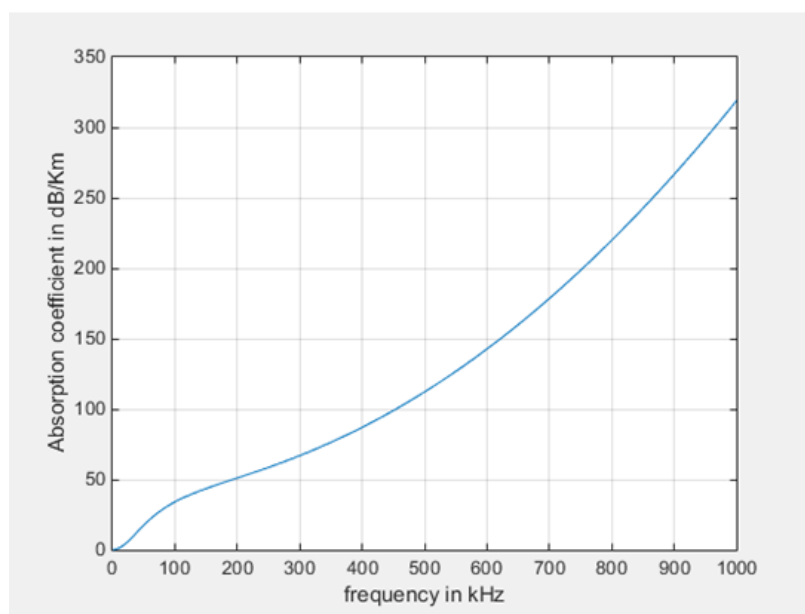

Fig.1 Absorption coefficient

\section{B. Multipath and Shadow zone}

Multipath effects are the fact that the acoustic signals experience different paths when arriving at the receiver, the paths may be affected by bottom and surface reflection or the combination of these two reflections (or other objects) in shallow water region, as described in Fig.2:

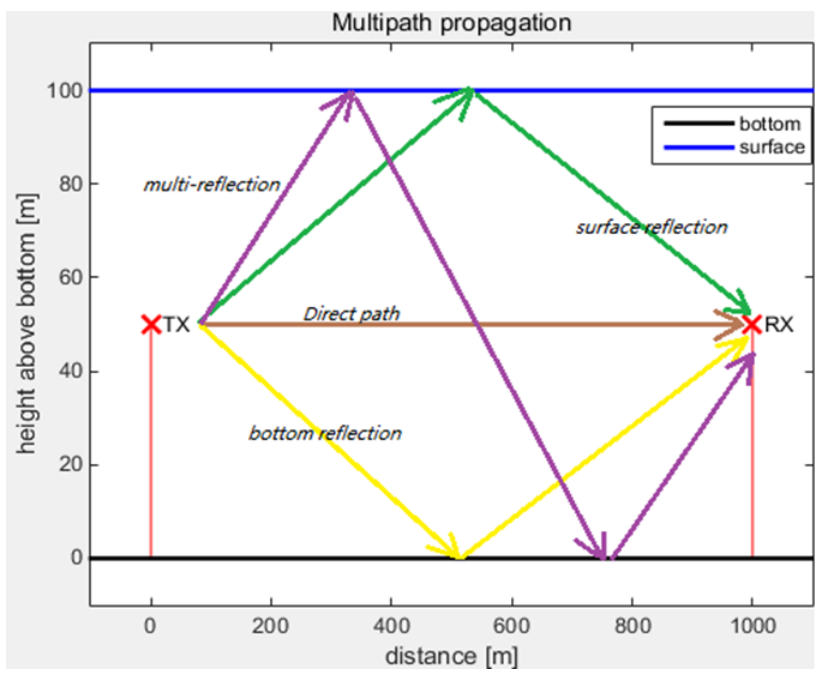

Fig. 2 Multipath effects in UAC system

A beam of transmitted sound rays may bend over or down due to refraction; however, these waves may avoid traveling through a specific region at the depth of approximately $80-100 \mathrm{~m}$, which called shadow zone.

\section{BPSK modulation}

we employ DS-BPSK modulation technique to modulate data bit in required frequency,Fig.3 show the block diagram of DSBPSK modulator:

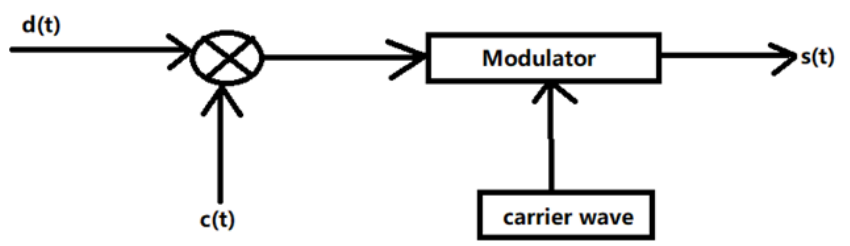

Fig.3 DS-BPSK modulator

From Fig.13,c(t) is spreading codes, we assume carrier frequency is $w 0$, therefore the transmitted signal $s(t)$ can be expressed as :

$$
\mathrm{s}(\mathrm{t})=\cos \left[w_{0} t+\emptyset_{c(t)}\right]
$$

where $\varnothing$ is phase modulation index, if we assume in PN sequences, when $\mathrm{c}(\mathrm{t})=0, \varnothing \mathrm{c}(\mathrm{t})=0$; when $\mathrm{c}(\mathrm{t})=1, \varnothing \mathrm{c}(\mathrm{t})=\pi$, BPSK modulation can be formed. However in practice, PN codes usually take polar properties which means $\mathrm{c}(\mathrm{t})=\{-1,1\}$, therefore the transmitted signal based on DS-BPSK modulation can be represented as:

$$
\mathrm{s}(\mathrm{t})=\mathrm{c}(\mathrm{t}) \cos \left[w_{0} t+\emptyset\right]
$$

If take data signal $d(t)$ into consideration, equation(2.18) can be expressed as:

$$
\mathrm{s}(\mathrm{t})=\mathrm{d}(\mathrm{t}) \mathrm{c}(\mathrm{t}) \cos \left[w_{0} t+\emptyset\right]
$$

Fig.4 shows the transmitted signal constellation after BPSK modulation(before spreading):

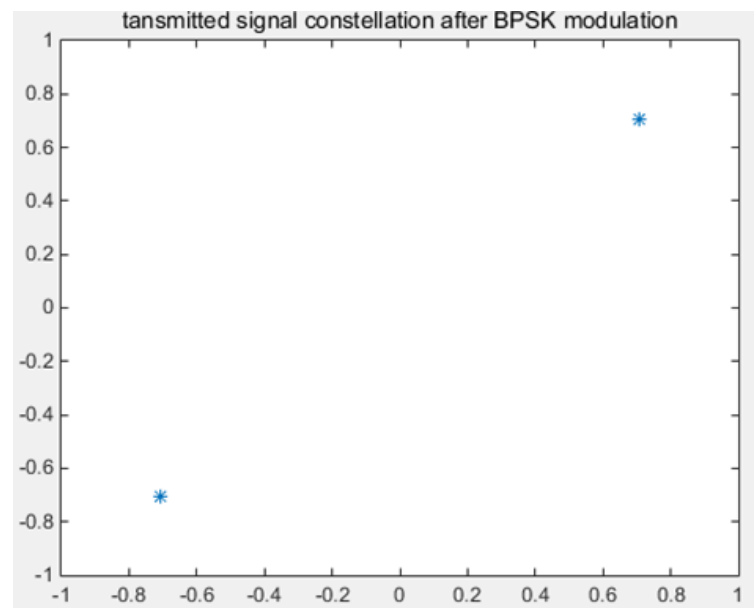

Fig.4 transmitted signal constellation after $\operatorname{BPSK}(\mathrm{SNR}=20 \mathrm{~dB})$

\section{D. m-sequences}

$\mathrm{M}$ sequences are wildly used as spreading codes due to its lowcomplexity generating method, which are born from a m-stage linear feedback shift register (LFSR). A sequence is generated by LFSR is called maximal sequences, simply m-sequence with the maximal period $\mathrm{Nc}=2^{\mathrm{m}}-1$ [6]. Fig.5 gives a 5 stages $\mathrm{m}$ sequences generator with a XOR operator: 


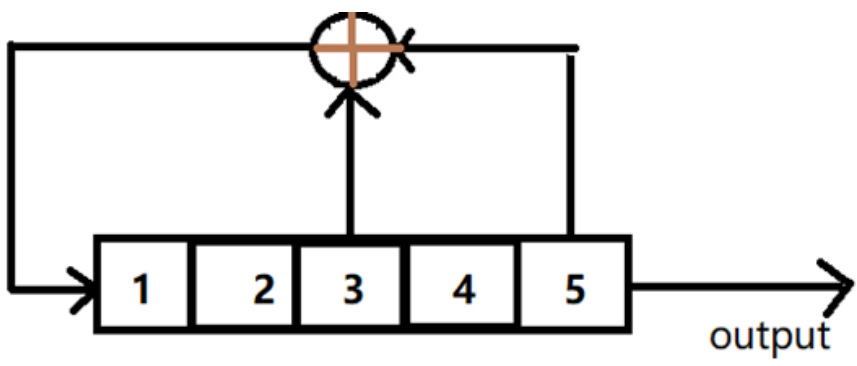

Fig.5 Five stages LFSR

As shown from Fig.7, the taps of feedback connection are determined by generator polynomial, in above case, the corresponding polynomial is:

$$
g(D)=D^{5}+D^{3}+1
$$

where $\mathrm{D}^{5}$ is the output of the fifth shift registers and $\mathrm{D}^{3}$ is the output of the third shift register, both of them are connected by modulo-2 adder and the result are fed back to the first shift register. The period is $2^{5}-1=31$, that value is also the spreading gain for DSSS system.

M-sequences are good at combating multipath effects due to they have excellent autocorrelation properties. The features of good autocorrelation are that the autocorrelation peak should be large and sharp, and the value of autocorrelation should approach zero for any time shift [7]. From Fig.6, we can find that the autocorrelation function is two valued, the peak value is 31 which is the length (or period) of the selected $\mathrm{m}$-seq, the plot is impulse-like.

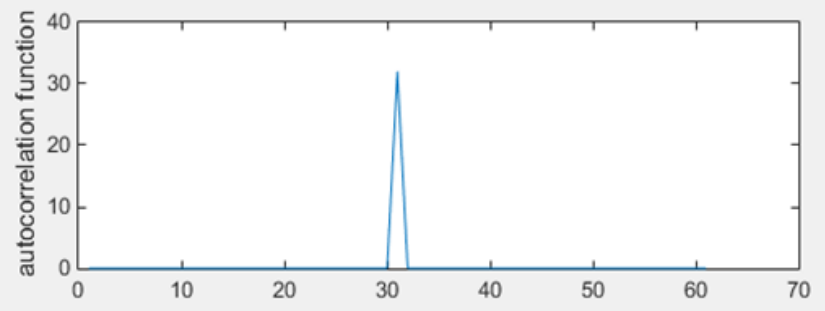

Fig. 6 autocorrelation function for m-seq. $\left(\mathrm{D}^{5}+\mathrm{D}^{3}+\mathrm{D}^{2}+1\right)$

However, the cross-correlation properties of $\mathrm{m}$-seq. is not good which degrading the system performance, and hence the smaller the cross correlation is, the better the interference is reduced among users. In order to select reasonable poor crosscorrelation between $\mathrm{m}$-seq pairs, the definition of preferred $\mathrm{m}$ seqs is proposed [7], as showed at below:

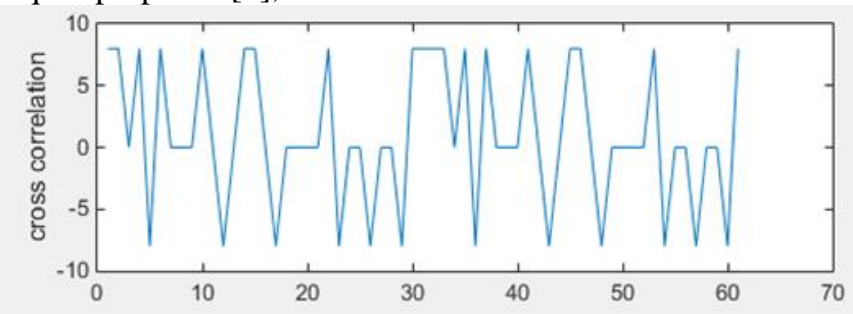

Fig. 7 cross-correlation function for preferred m-seqs.

From Fig.7, the preferred m-seqs have three cross-correlation values. By contrast, it will be called non-preferred, If It exhibit noise-like cross-correlation (may multiple valued), in particular the peaks may higher than preferred version.

\section{Gold codes}

The objective of inventing gold sequences is to get a lower cross-correlation at the expense of improving a little autocorrelation. The code is generated from a pair of $\mathrm{m}$-sequences with equal period by modulo- 2 adding in a different phase shifts [7]. The structure of gold code generator is illustrated as below:

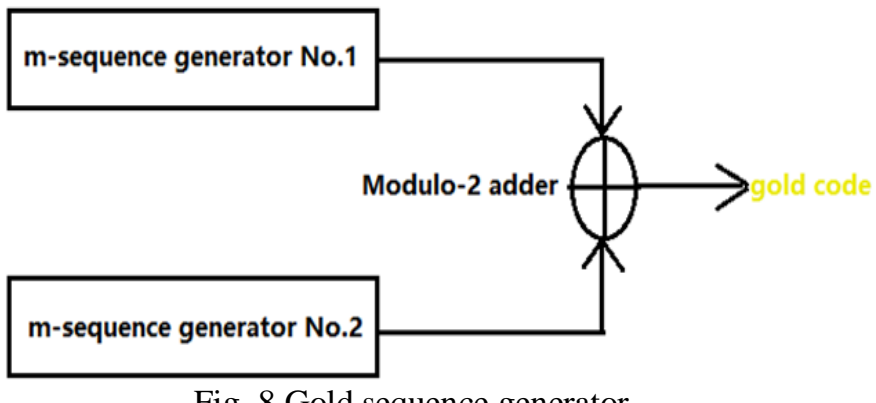

Fig. 8 Gold sequence generator

The key operation for producing gold code is to ensure there are $\mathrm{Nc}=2^{\mathrm{m}}-1$ different relative phase shift, and thus the number of different gold codes can be produced during this process is $2^{\mathrm{m}}+1$ [7]. The autocorrelation function of gold code is showed in Fig.9:

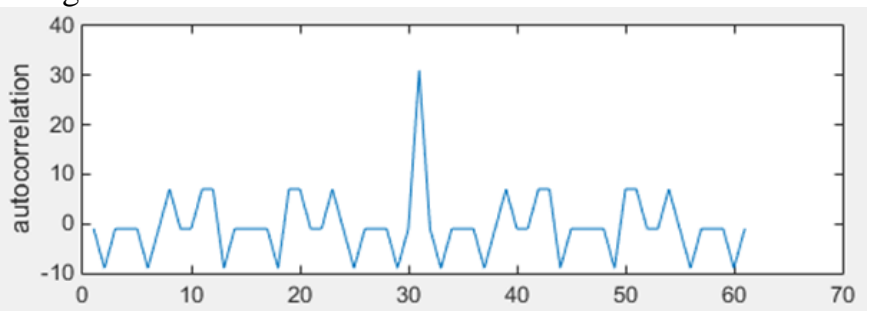

Fig.9 Autocorrelation function for gold codes

From Fig.9, there is an obviously sharp peak with maximal value equal 31 because the gold code is generated by preferred 5-degree m-sequences as described in Fig.5, but the side lobe is not flat which is actually three valued, therefore we can conclude that the autocorrelation proprieties is not as good as $\mathrm{m}$-sequences.

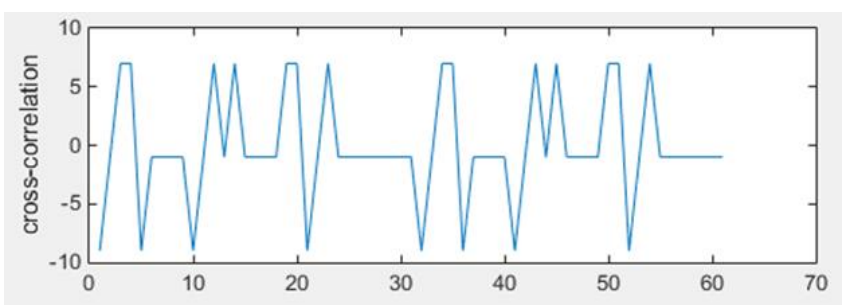

Fig.10 Gold code cross-correlation function

From Fig.10, the feature of cross-correlation for gold code is the same with $\mathrm{m}$-sequence because they are three valued, however compared with Fig.7, the gold code has a slightly lower cross-correlation than m-sequences, and thus we can conclude that gold codes arrive a balance value(or level) 
between cross-correlation and autocorrelation. Therefore gold codes is good to reduce the interference from other users at receiver in multi-users CDMA system.

\section{E. Channel model}

we would first implement the system in AWGN (additive white Gaussian noise) and in LTI plus AWGN channel model respectively. AWGN is a basic channel model widely used in communication simulation, it can be expressed as below:

$$
\mathrm{r}(\mathrm{t})=\mathrm{s}(\mathrm{t})+\mathrm{n}(\mathrm{t})
$$

where $r(t)$ is the received signal which contains the noise element, $s(t)$ is the transmitted signal, $n(t)$ is white noise. As it name described in computing platform e.g. MATLAB, it can be involved by one-shot addition.

LTI channel model can be essentially created by a FIR filter, which is multipath (frequency -selective) channel model, in mathematical way it is presented as below [8]:

$$
\mathrm{x}(\mathrm{t})=\sum_{i=0}^{L-1} a_{i} s\left(t-\tau_{i}\right)
$$

where $\mathrm{L}$ is the number of resolvable paths, $\mathrm{x}(\mathrm{t})$ is the received signal, $s(t)$ is the transmitted signal with short pulse, ai are complex path gains, $\tau_{\mathrm{i}}$ are time delays, the structure is showed in Fig.11:

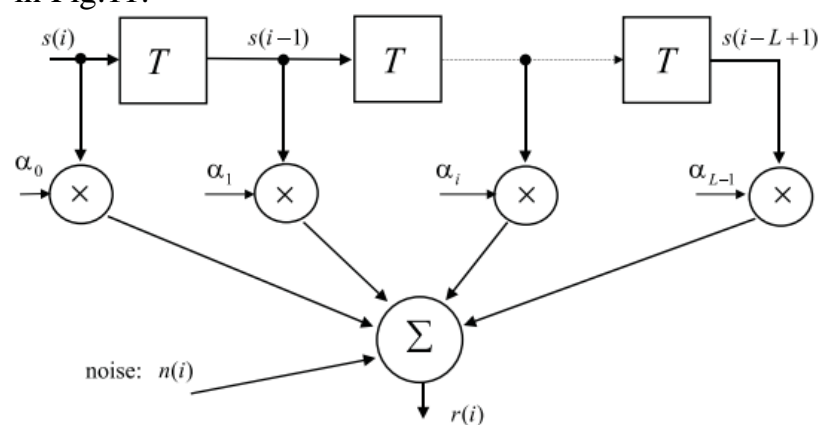

Fig. 11 LTI channel model by FIR filter
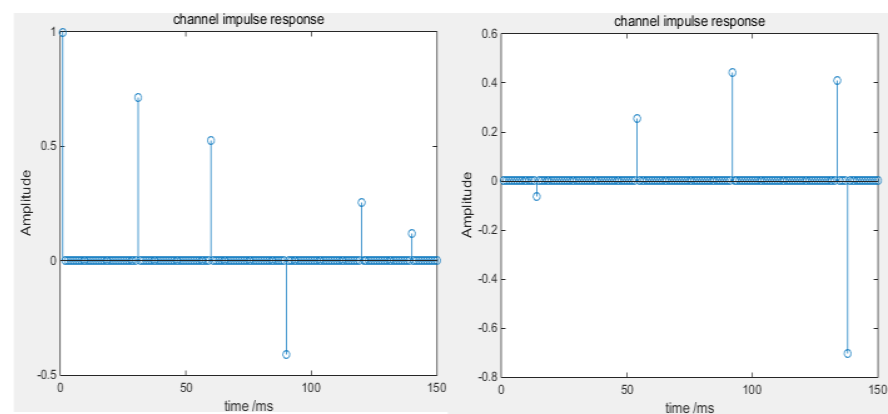

Fig. 12 Channel model 1(left) and channel model 2(right)

Fig. 12 shows two channel model created by FIR filter, the taps of the channel in left case is six, in right is five, the features of impulse response are different between channel one and two. For example, in channel one, the signal can direct arrive receiver through the first path, the maximum time delay is approximately $145 \mathrm{~ms}$, therefore we can calculate the time spread: max(time delay) minus min(time delay), in this case, it is $145 \mathrm{~ms}$, that is a critical characteristic of multipath channel.

\section{SimUlation RESUltS}

Fig. 13 show the structure of the modem:

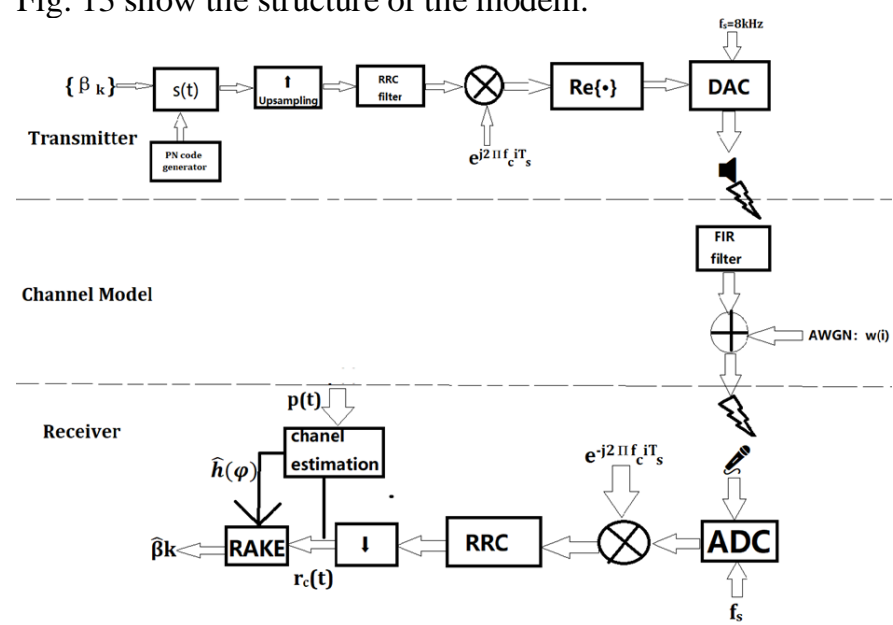

Fig.13 Structure of designed modem

From Nyquist sampling theorem, we set sampling rate fs $=10^{6} \mathrm{~Hz}$ which should be two times larger than frequency bandwidth $2000 \mathrm{~Hz}$ at least, in this case, it is enough.Fig. 14 and Fig. 15 shows the transmitted signal waveform in time domain and its power spectrum density after pulse shaping respective as below:

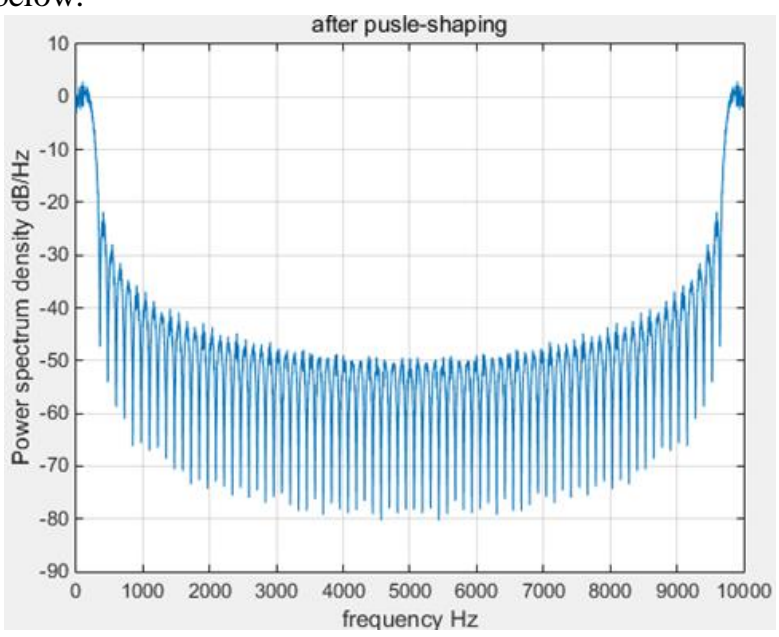

Fig. 14 Power spectrum density after pulse shaping

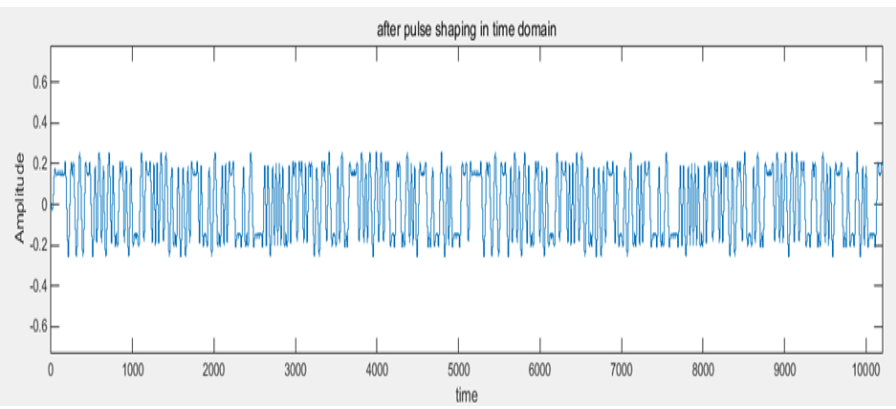

Fig. 15 Signal waveform after pulse shaping in time domain 
After RF modulation, the central frequency is shifted from zero to the required frequency:

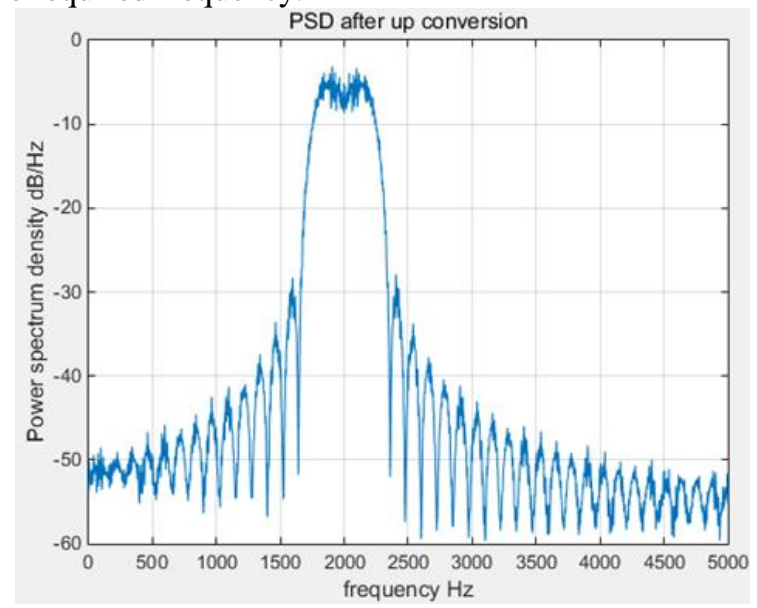

Fig. 16 PSD after up conversion

From Fig.16, the central frequency is the carrier frequency as we set $\mathrm{fc}=2 \mathrm{kHz}$.

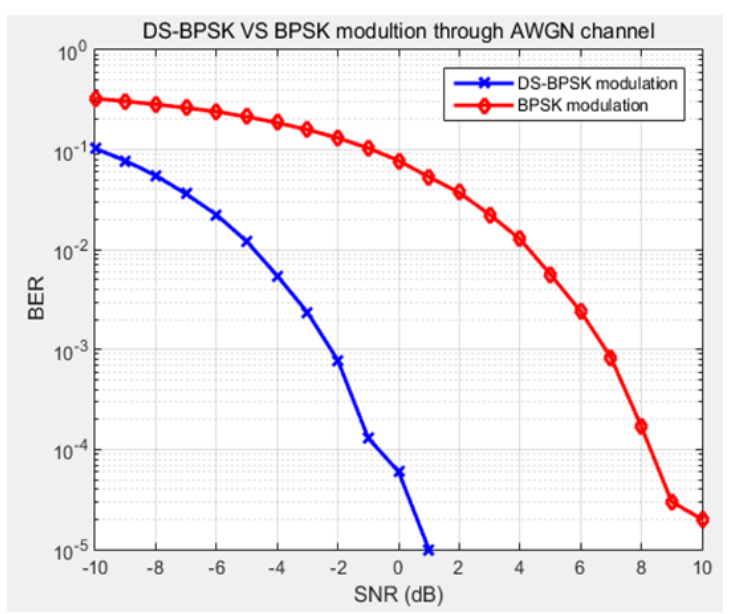

Fig. 17 DS-BPSK detection performance under white noise

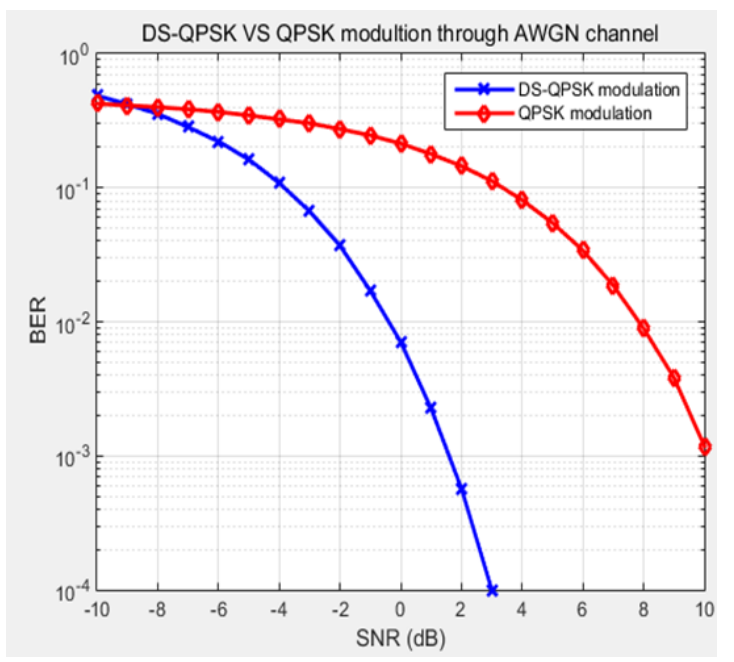

Fig. 18 DS-QPSK detection performance under white noise Fig.17and Fig. 18 shows the simulation result of if DS based MPSK has a positive or negative affect to the communication system at different SNR under AWGN channel. From the BER curves, we can find that the bit error rates will decrease with the increasing of SNR under BPSK, QPSK, DS-BPSK and DSQPSK modulations, but it is obviously that DS-BPSK and DSQPSK provide a lower bit error level than without using spread spectrum techniques. The detection benefit is caused by the operation of spread system bandwidth, therefore the ability of combating interference is enhanced. (use 3-degree $m$ sequences, period=7)

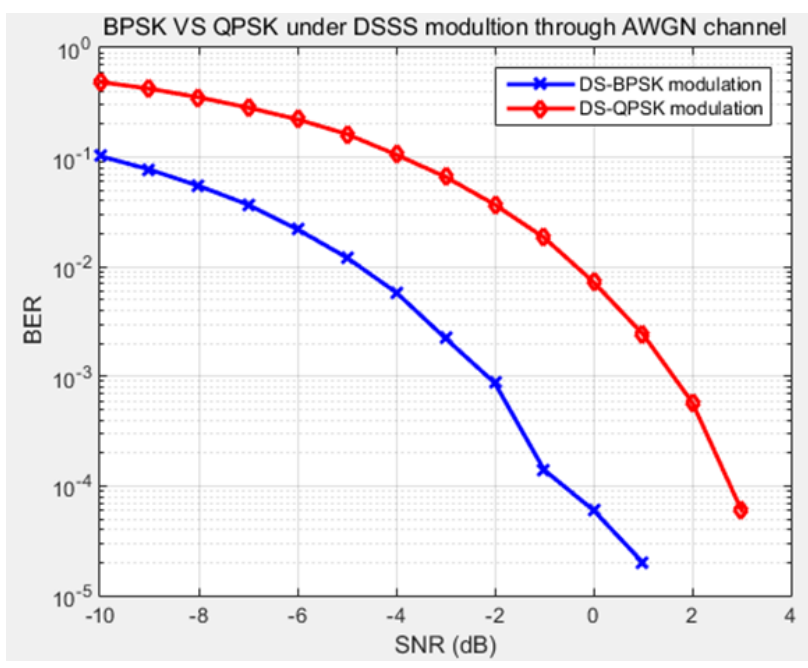

Fig.19 BER simulation under QPSK and BPSK modulation

The bandwidth efficiency for QPSK is higher than BPSK in classic communication modem according to the previous experience, however from Fig. 19, DS-BPSK modulation has a good detection performance than DS-QPSK, which means the benefit for QPSK has lost in DSSS system.

In next experiment, we upgrade the channel model to LTI channel which established by FIR filter, also plus white noise (AWGN), we choose m-sequences with period 127 to multiply the input bit, at receiver side the taps of the rake receiver are five, the taps location depends on the channel estimation, full parameter settings as below table:

\begin{tabular}{|l|r|}
\hline PN code: & M-sequence \\
\hline Processing gain: & 127 \\
\hline Channel taps & 5 \\
\hline Channel model & LTI+AWGN \\
\hline Rake taps & 5 \\
\hline Diversity schemes & MRC \\
\hline Roll-off factor & $0.22($ UMTS) \\
\hline Carrier frequency & $2000 \mathrm{~Hz}$ \\
\hline Sampling frequency & $10000 \mathrm{~Hz}$ \\
\hline symbol rate & $500 \mathrm{~Hz}$ \\
\hline OSF & 20 \\
\hline
\end{tabular}




\section{International Journal of Engineering Applied Sciences and Technology, 2021 \\ Vol. 5, Issue 9, ISSN No. 2455-2143, Pages 50-57 \\ Published Online January 2021 in IJEAST (http://www.ijeast.com)}

Table -1 parameter settings

Fig.20 shows the performance of Rake receiver:

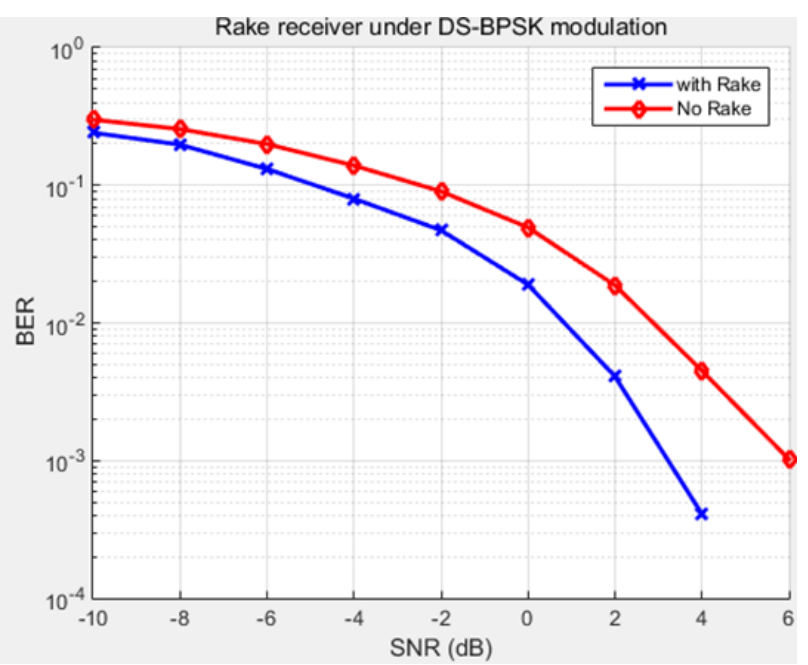

Fig.20 if rake can improve the performance of DS-BPSK based system

From Fig.20, when DS-BPSK system employ rake receiver to achieve diversity reception, the BER is lower than without rake, therefore we can conclude that Rake receiver has good capability to process multipath signals. Multipath affects are a disadvantage for conventional receiver; however, rake can utilize the drawback to become a benefit which achieved by combining multipath signals energy into a stronger one at the output to optimize SNR.

The following simulations are mainly based on the parameters in Tab.3, we only change a specific element setting, for example, in this case, the kinds of PN code are compared.

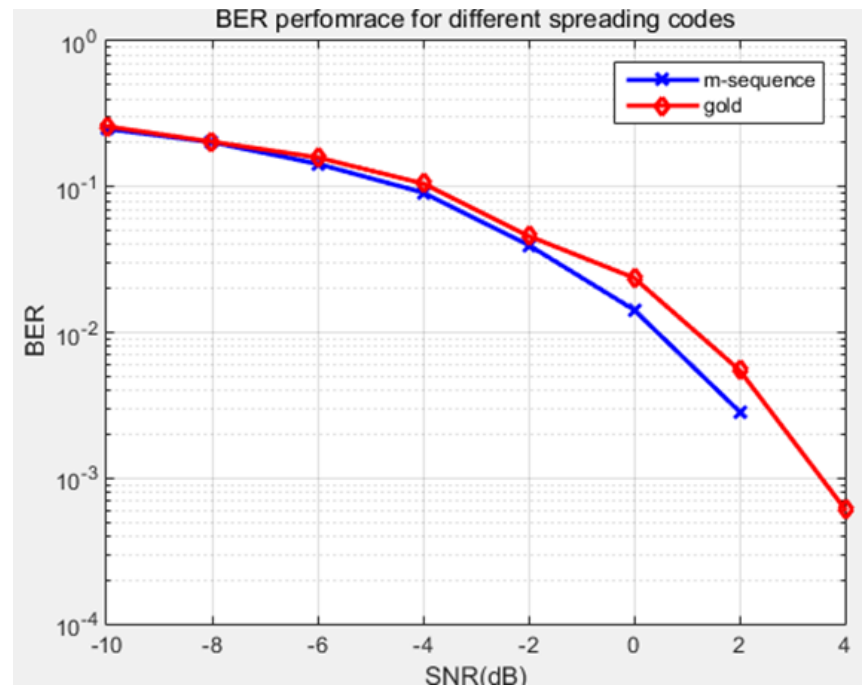

Fig. 21 Comparison of m-sequence and gold code

In Fig.21, the difference of PN codes BER performance between $\mathrm{m}$-sequence and gold code is not obviously, in this case, the BER level of m-sequence is slightly lower than gold code's BER curve.

We only change the period of the m-sequences to compare the simulation results as below:

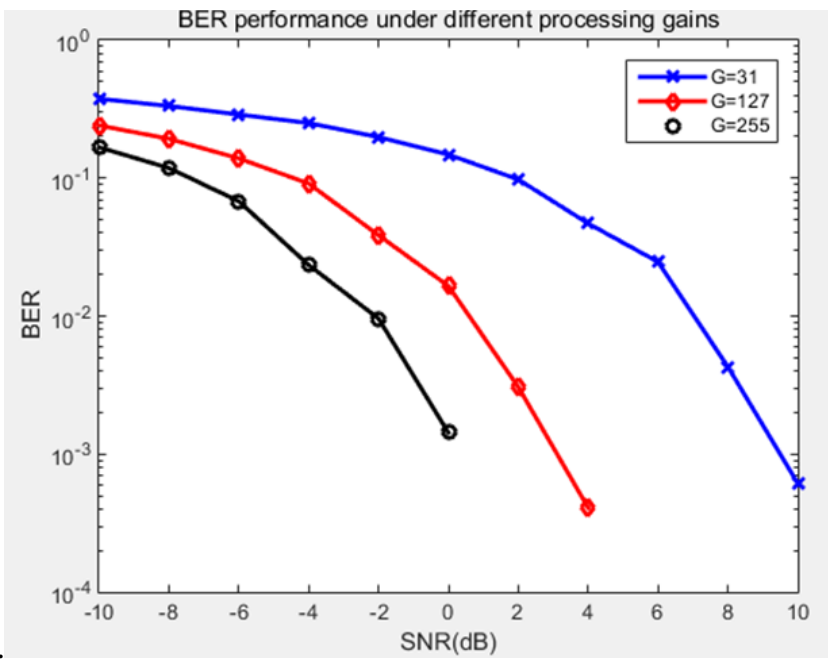

Fig.22 BER curves under G=31,127,255

In Fig.22, when the processing gain increased, the BER performance will be improved, within this simulation, we can draw the conclusion that the detection performance will be better with the increasing of PN code period.

When rake filter processes the multipath signals, the number of its arms would affect the detection performance, Fig. 23 show the BER curves under different number of Rake taps which adopting MRC algorithm at the output:

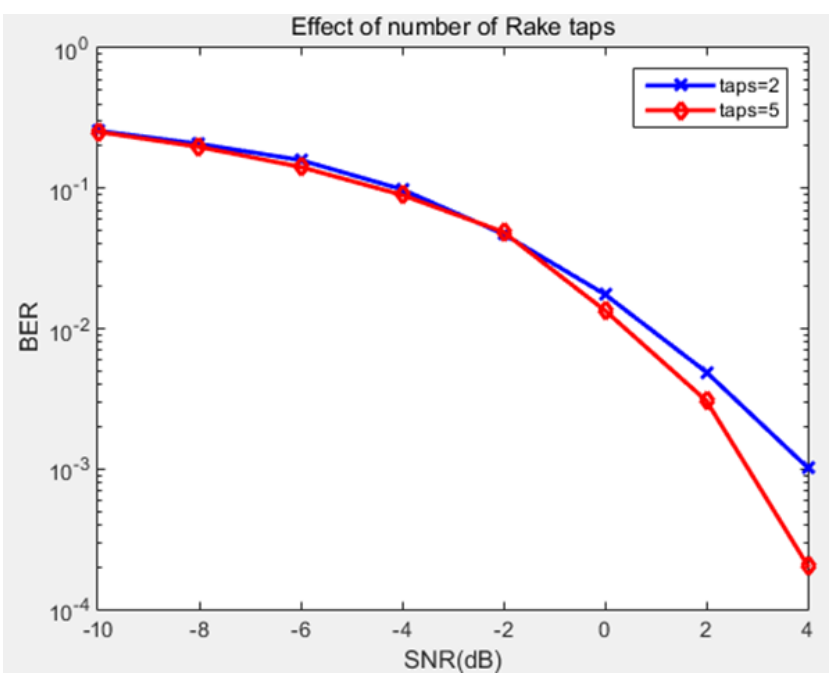

Fig. 23 system performance affected by finger numbers

In Fig.23, as the increasing of fingers, the BER level will decrease, which means rake with more taps can utilize and combine more signal energy from multipath channel, therefore the output SNR is optimized [14]. However, the arms number decide the complexity level of the system, The more arms 
number, The more complexity for hardware modem, The more overhead. We are interested in seeking a trade-off between the system performance, cost and complexity when choosing rake taps [15]. In addition, the detection performance may not be improved with continuously increasing the number of arms, a required number is: $\mathrm{D}=\tau_{\max } / \mathrm{T}_{\mathrm{c}}+1$ [9].

At output stage of rake receiver, adopting different combining techniques would present different detection performance which depend on their algorithm thinking. We artificially involve the ISI element, and delay the multipath signals by our interest as below:
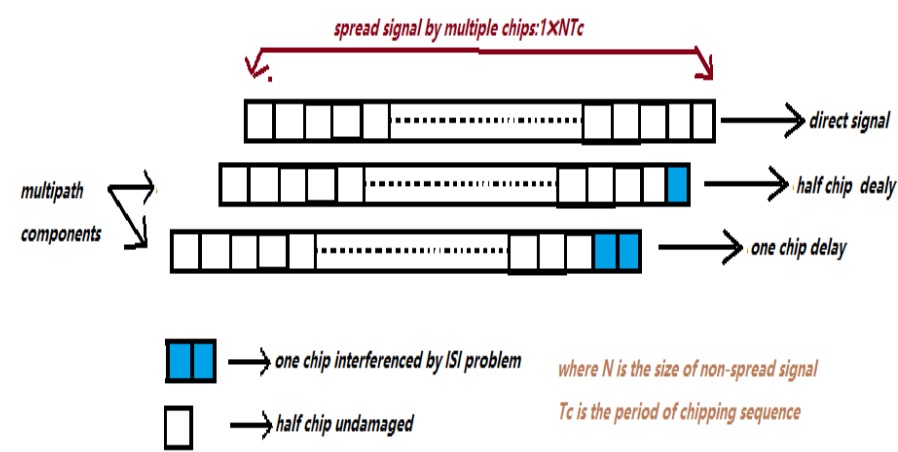

Fig. 24 Multipath signals with ISI element

From Fig.24, the multipath components are consisted of three version of signals, the direct signal is neither delayed or interference by ISI [10]. The other two signals are delayed and affected by their corresponding parameters, we set the path gain at $-1,-2$ and $-5 \mathrm{~dB}$ for $1 \mathrm{st}, 2 \mathrm{nd}$ and 3 th signal respectively. In this simulation we try a new PN code-Walsh code with processing gain 16. The result is illustrated by Fig. 25:

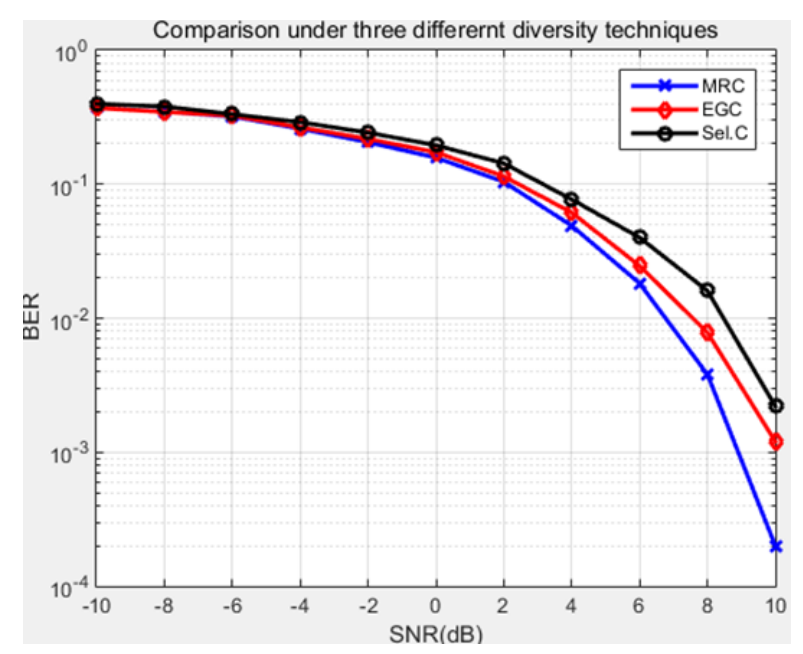

Fig. 25 BER performance by three kinds of diversity techniques

From Fig.25, MRC technique achieves a lower BER performance compared with other techniques, especially with the increasing of SNR, the benefit is more obviously.
In air acoustic channel, when the transmitter and receiver keep a relative shift, the detection quality will be affected by the frequency shift, however in UAC system [11], the Doppler effect may severer than in radio communication due to the complex-dynamic underwater channel, therefore employing low-complexity and good performance Doppler estimation algorithm is necessary to compensate the frequency shift at receiver [13].

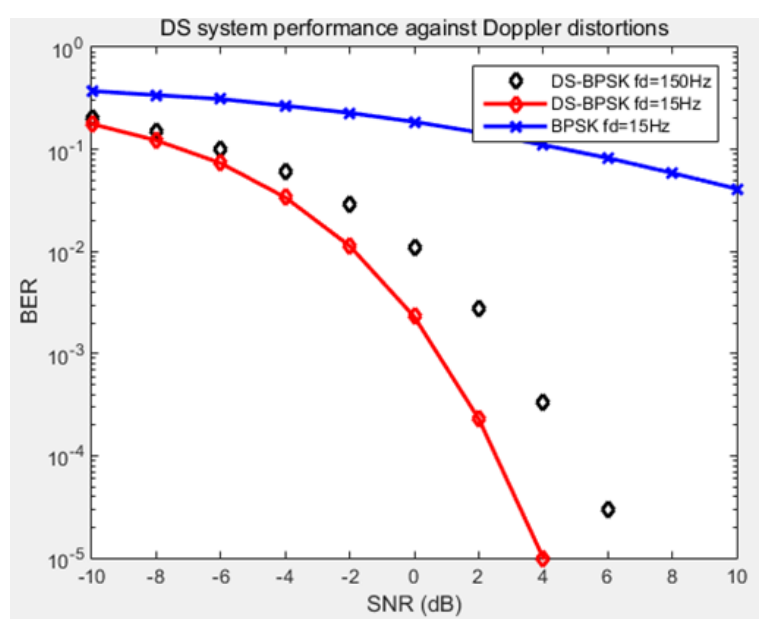

Fig. 26 Comparison of DS-BPSK and BPSK for frequency shift

In Fig.26, It shows that DS-BPSK modulation has a better BER performance than conventional BPSK modulation which means that DSSS system has the ability to resist frequency shift, however it also needs frequency offset algorithm to do further improvement [12].

\section{CONCLUSION}

In this paper we have done nine simulations under different circumstances, therefore we can get some hints when designing the real DSP platform for UAC system. For example, if we do not consider the cost e.g., power, efficiency and money, we can choose DS-BPSK modulation with rake receiver (MRC) to create the modem, especially with higher period of the selected chipping sequence, we can also increase the receiver fingers aiming to search more multipath signal energy.

\section{REFERENCE}

[1] C. Liu.(2011).“Advanced Signal Processing Techniques for Underwater Acoustic Communication Networks," University of York, Phd thesis.

[2] P. S. Duke.(2002)."DIRECT-SEQUENCE SPREADSPECTRUM MODULATION FOR UTILITY PACKET TRANSMISSION IN," UNITES STATES Naval Postgraduate School, California.

[3] J. Ribas.(2009). "Underwater Wireless Video Transmission using Acoustic OFDM," Massachusetts Institute of Technology, Massachusetts. 
[4] J. Proakis, M. Stojanvic and E. Sozer.(2000). "Underwater Acoustic Networks," IEEE JOURNAL OF OCEANIC ENGINEERING, vol. 25, no. 1, pp. 72-83.

[5] Y. Lysanov and L. Berkhovskikh.(1982).Fundamentals of Ocean Acoustics, New York: Springer.

[6] M. Suzuki and S. Sasaki.(1992)."Digital acoustic image transmission system for deep sea," Proc.OCEANS'92, pp. 567-570.

[7] M. Stojanovic, J. A. Catpiovic and J. G. Prokais.(1993).“Adaptive multichannel combining," Journal of the Acoustical, vol. 94, pp. 1621-1631.

[8] G. Gussen, P. Diniz, M. Campos, M. Martins, F. Costa and J. Gois.(2016).“A Survey of Underwater Wireless Communication Technologies," Journal of Communication andInformationSystems, vol. 31, no. 1, pp. 242-255.

[9] K. Fazel and S. Kaiser.(2003).Multicarrier and Spread spectrum systems, Germany: Wiely.

[10] M. Stojanovic and J. Preisig.(2009). "Underwater Acoustic Communication Channels:Propagation Models and," IEEE Communications Magazine, pp. 84-89.

[11] C. Liu, Y. Zakharov and T. Chen.(2012)."Doubly Selective Underwater Acoustic Channel," IEEE TRANSACTIONS ON VEHICULAR TECHNOLOGY, vol. 61, no. 3, pp. 938-950.

[12] Y. Zhang, A. Wang and B. Li.(2018)."Underwater Acoustic Channels Characterization for Underwater Cognitive Acoustic Networks," International Conference on Intelligent Transportation, Big Data \& Smart City, pp. 223-226.

[13] E. M. Sozer, J. G. Proakis, M. Stojanovic, M. Hatch, J. A. Rice and A. Benson.(2002)."Direct Sequence Spread Spectrum Based Modem for Under,” IEEE, pp. 228-233.

[14] I. Martoyo, Philip, E. Wijanto, H. Kanalebe and K. Gandi.(2010)."Chaos Codes vs. Orthogonal Codes for CDMA,” ISSSTA2010, pp. 17-20.

[15] H. Liu.(2000). Signal processing applications in CDMA communications, Boston: Artech House Publishers. 